Supporting Information

\title{
Surface Sensitive Photoluminescence of Carbon Nanodots: Coupling between the Carbonyl Group and $\pi$-Electron System
}

Cui Liu, ${ }^{\dagger,}$ Lei Bao, ${ }^{\dagger}$ Mengli Yang, ${ }^{\dagger}$ Song Zhang, ${ }^{*,, "}$ Miaomiao Zhou, ${ }^{\ddagger}, "$ Bo Tang, ${ }^{\dagger}$ Baoshan Wang, ${ }^{\dagger}$ Yufei Liu, ${ }^{\S}$ Zhi-Ling Zhang, ${ }^{,, \dagger}$ Bing Zhang, ${ }^{\ddagger}, "$ and Dai-Wen Pang $\dagger$

${ }^{\dagger}$ Key Laboratory of Analytical Chemistry for Biology and Medicine (Ministry of Education), College of Chemistry and Molecular Sciences, State Key Laboratory of Virology, The Institute for Advanced Studies, and Wuhan Institute of Biotechnology, Wuhan University, Wuhan 430072, P. R. China

${ }^{\ddagger}$ State Key Laboratory of Magnetic Resonance and Atomic and Molecular Physics, Wuhan Institute of Physics and Mathematics, Chinese Academy of Sciences, Wuhan 430071, P. R. China

$\S$ Key Laboratory of Optoelectronic Technology \& Systems (Ministry of Education), Centre for Intelligent Sensing Technology, College of Optoelectronic Engineering, Chongqing University, Chongqing 400044, P. R. China

" University of Chinese Academy of Sciences, Beijing 100049, P. R. China

Materials: Carbon fiber powder were purchased from Shanghai Nengkang Tansu Technology Co., Ltc.. $\mathrm{HNO}_{3}(65 \% \sim 68 \%), \mathrm{NaHCO}_{3}$ (AR), $\mathrm{NaOH}(\mathrm{AR})$ and $\mathrm{NaBH}_{4}$ (AR) were purchased from local supplies. The ultrapure water used in all experiments was purified using a Synergy ultrapure water system (Millipore).

Synthesis of the initial C-dots: carbon fiber powder $(0.4 \mathrm{~g}, 500 \mathrm{Mesh})$ were added to a boiled $\mathrm{HNO}_{3}$ solution $(10 \mathrm{M}, 30 \mathrm{~mL})$ and refluxed for $4 \mathrm{~h}$. The resulting solution was 
neutralized with $\mathrm{NaHCO}_{3}$ and filtered by $0.22 \mu \mathrm{m}$ membrane filters. The solution containing C-dots was dialyzed for 7 days and then ultrafiltered through Millipore centrifugal filter devices. Finally, the C-dots $(<3 \mathrm{kDa})$ were collected and investigated.

Preparation of C-dots- $\mathrm{NaBH}_{4}: 20 \mathrm{mg}$ of the initial C-dots was added to $50 \mathrm{~mL}^{\text {of } \mathrm{NaBH}_{4}}$ solution $(5 \mathrm{M})$ and stirred for $48 \mathrm{~h}$ at room temperature. The resulting solution was neutralized with $\mathrm{HCl}$, and then dialyzed for 3 days.

Preparation of $\mathrm{C}$-dots- $\mathrm{NaOH}$ and $\mathrm{C}$-dots- $\mathrm{NaBH}_{4}-\mathrm{NaOH}$ : $10 \mathrm{mg}$ of C-dots (or C-dots$\mathrm{NaBH}_{4}$ ) and $2 \mathrm{~g}$ of $\mathrm{NaOH}$ were added to $10 \mathrm{~mL}$ of water and then heated at $200{ }^{\circ} \mathrm{C}$ for $24 \mathrm{~h}$. The resulting solution was neutralized and then dialyzed for 3 days.

Preparation of $C$-dots-HI: The mixture of the initial C-dots $(10 \mathrm{mg}), 57 \% \mathrm{HI}(2 \mathrm{~mL})$ and acetic acid $(20 \mathrm{~mL})$ was refluxed for $24 \mathrm{~h}$, and then poured into $25 \mathrm{~mL}$ of sodium bisulfite solution. The resulting mixture was then dialyzed for three days.

Characterization: Transmission electron microscopy (TEM) images of C-dots were observed on a Hitachi HT7700 transmission electron microscope at $80 \mathrm{kV}$. The PL and FT-IR spectra were performed by a Horiba Jobin Yvon Fluorolog-3 fluorescence spectrometer and a Thermo Nicolet 360 FT-IR spectrophotometer, respectively. The XPS spectra were recorded using a VG Multilab 2000 X-ray photoelectron spectrometer. The Raman spectra were performed by a Renishaw InVia Confocal Raman Spectroscopy. The proton magnetic resonance $\left({ }^{1} \mathrm{H}\right.$ NMR) spectra were recorded at $400 \mathrm{MHz}$ on a Bruker Ascend ${ }^{\mathrm{TM}} 400$ spectrometer using $\mathrm{D}_{2} \mathrm{O}$ as solvent.

The quantum yield $(Q Y)$ of $C$-dots: The quantum yields of C-dots were measured by using comparative method $\left(\varphi_{\mathrm{x}}=\varphi_{s t} \frac{K_{x}}{K_{s t}} \frac{\eta_{x}^{2}}{\eta_{s t}^{2}}\right)$. The reference standard is fluorescein (free acid, in $0.1 \mathrm{M} \mathrm{NaOH}, \varphi_{\mathrm{st}}=95 \%$ ). In the equation, $\varphi, \mathrm{K}$, and $\eta$ are the QY, slope from the plot of integrated PL intensity versus absorbance, and the refractive index of the solvent, 
respectively. The subscripts st and $\mathrm{x}$ are standard and test samples, respectively. The QYs of the four kinds of C-dots were measured in $0.1 \mathrm{M} \mathrm{NaHCO}_{3}$ solution.

Theoretical calculations: The C-dots structures were subjected to extensive geometrical relaxation and annealing with the COMPASS force-field. ${ }^{1}$ The conformation with the lowest energy was further optimized using the semi-empirical PM6 Hamiltonian. ${ }^{2}$ The aqueous environment was considered using the numerical self-consistent reaction field method with the conductor-like screening model. ${ }^{3}$ The electronic levels and the corresponding oscillator strength were calculated using the configuration interaction with all single excitations. ${ }^{4}$

The TA spectra: Ultrafast broadband absorption measurements were performed based on a Ti:sapphire femtosecond laser system. Details of the femtosecond laser system have been described elsewhere. ${ }^{5}$ Briefly, the seed beam is generated by a commercial Ti:sapphire oscillator pumped by a CW second harmonic of an Nd:YVO4 laser, and then amplified by an $\mathrm{Nd}: Y L F$ pumped regenerative amplifier to generate a $1 \mathrm{kHz}$ pulse train centered at $800 \mathrm{~nm}$ of approximately $35 \mathrm{fs}$ pulse width and with maximum energy of $1 \mathrm{~mJ} /$ pulse. Excitation light at $400 \mathrm{~nm}$ is the second harmonic generation of the fundamental pulse obtained by a $1 \mathrm{~mm}$ thick BBO crystal. The excitation intensity was set to less than $5 \mu \mathrm{J} /$ pulse for measurements. A white light continuum generated by focusing the fundamental light at $800 \mathrm{~nm}$ on a $1 \mathrm{~mm}$ sapphire plate is reflected from the front and back surfaces of a quartz plate to obtain the probe and reference beams. The pump and probe pulses intersect in the sample at an angle of $\sim 4^{\circ}$, and the reference beam is transmitted through the sample at a different spot. The relative polarization of the pump and probe pulses is set to the magic angle for all the measurements. A linear translation stage is used to delay the probe beam to monitor the pump-probe dynamics. The resulting spectra are detected by a CCD camera (PI-MAX, 1024×256 pixel array) equipped with a spectrometer (Princeton, SpectraPro 2500i). The instrumental response function of the system, determined by cross correlation between the excitation and probe pulses using the optical Kerr-gate method, is typically better than 250 fs. 


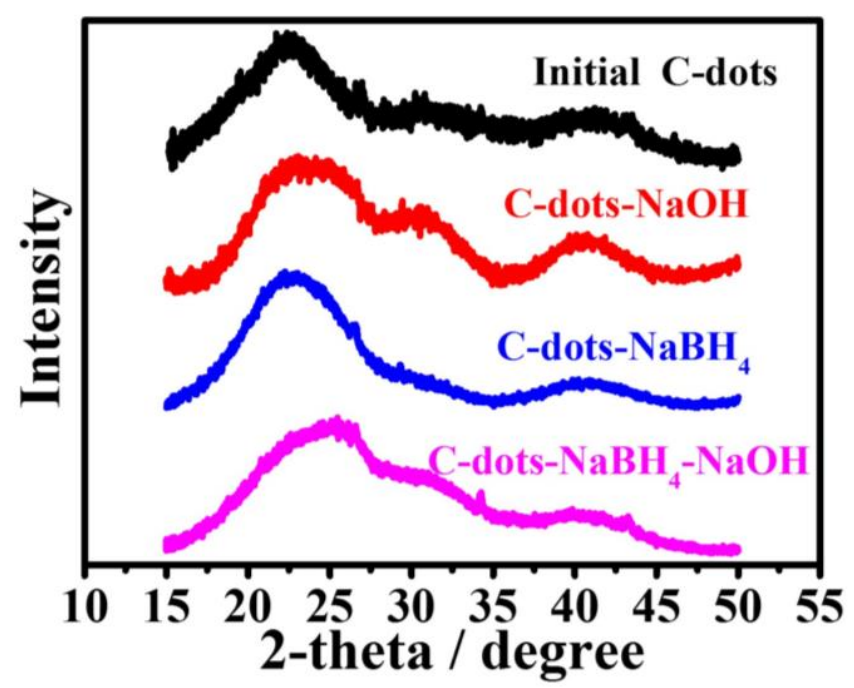

Figure S1. XRD patterns of the initial C-dots, C-dots- $\mathrm{NaOH}$, C-dots- $\mathrm{NaBH}_{4}$, and C-dots$\mathrm{NaBH}_{4}-\mathrm{NaOH}$.

The XRD patterns of C-dots before and after reduction by $\mathrm{NaBH}_{4}$ did not change. After a hydrothermal route in $5 \mathrm{M} \mathrm{NaOH}$ solution, the $2 \theta$ values in the range of $22-25^{\circ}$ of C-dots$\mathrm{NaOH}$ and C-dots- $\mathrm{NaBH}_{4}-\mathrm{NaOH}$ broadened slightly.
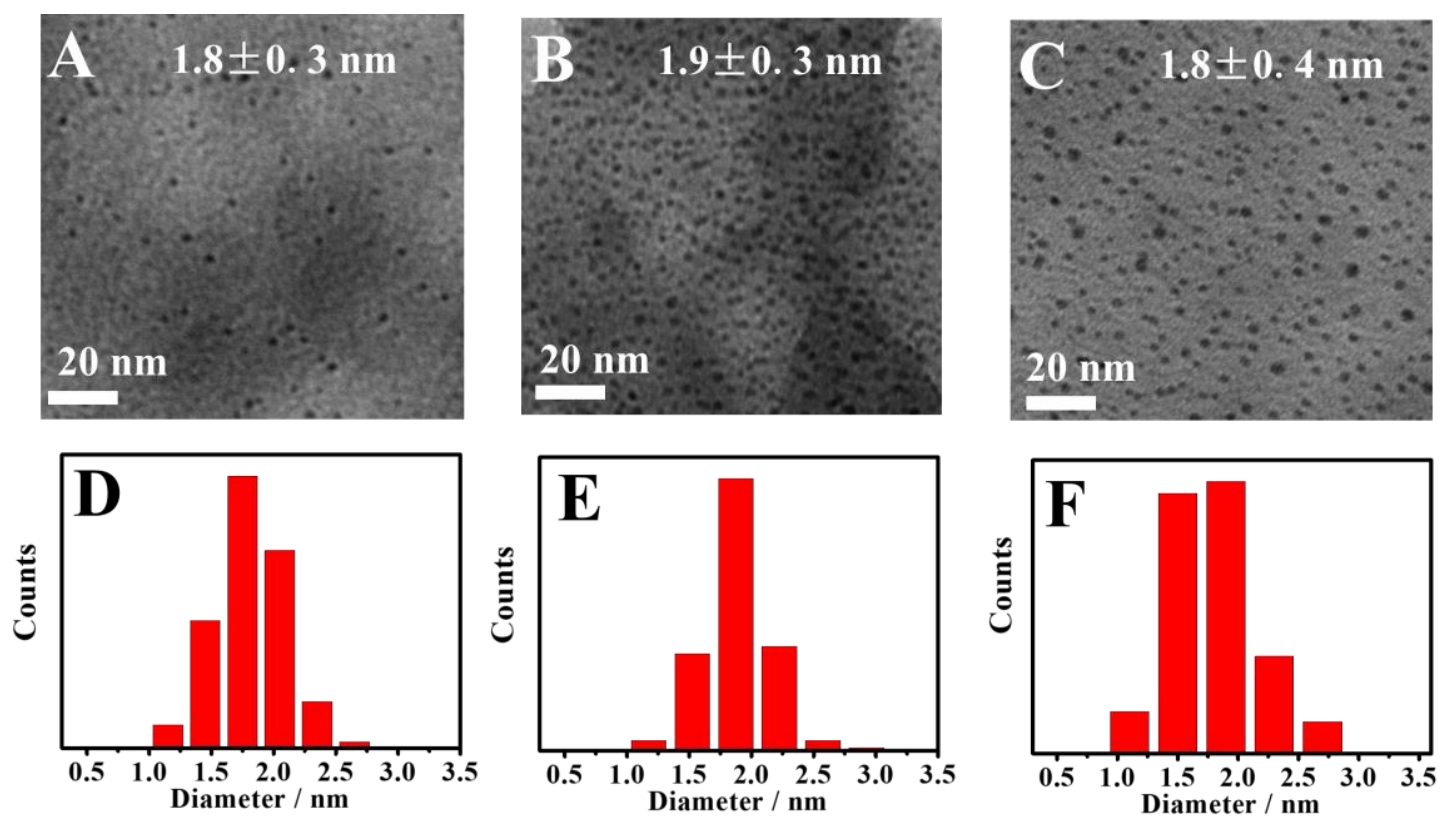

Figure S2. The TEM images and corresponding size distributions of (A, D) C-dots- $\mathrm{NaBH}_{4}$, (B, E) C-dots- $\mathrm{NaOH}$, and (C, F) C-dots- $\mathrm{NaBH}_{4}-\mathrm{NaOH}$. 


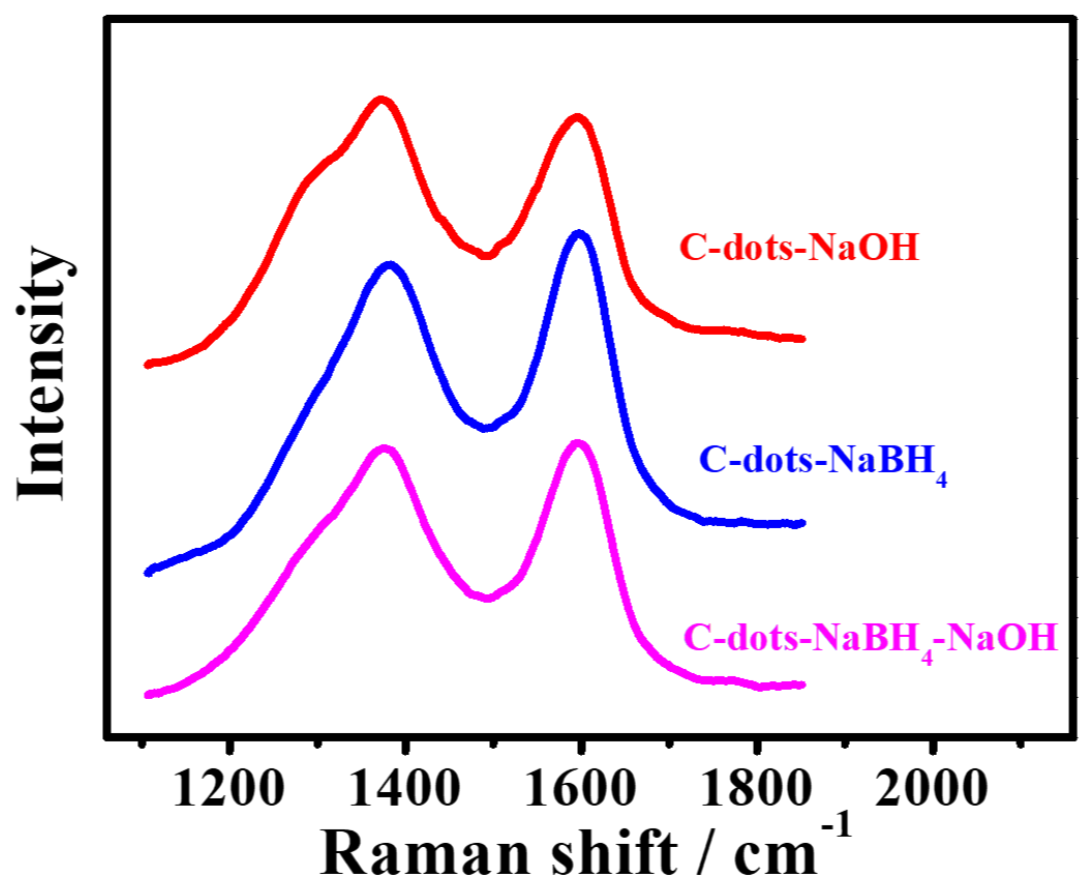

Figure S3. Raman spectra of the C-dots- $\mathrm{NaOH}, \mathrm{C}$-dots- $\mathrm{NaBH}_{4}$, and C-dots- $\mathrm{NaBH}_{4}-\mathrm{NaOH}$.
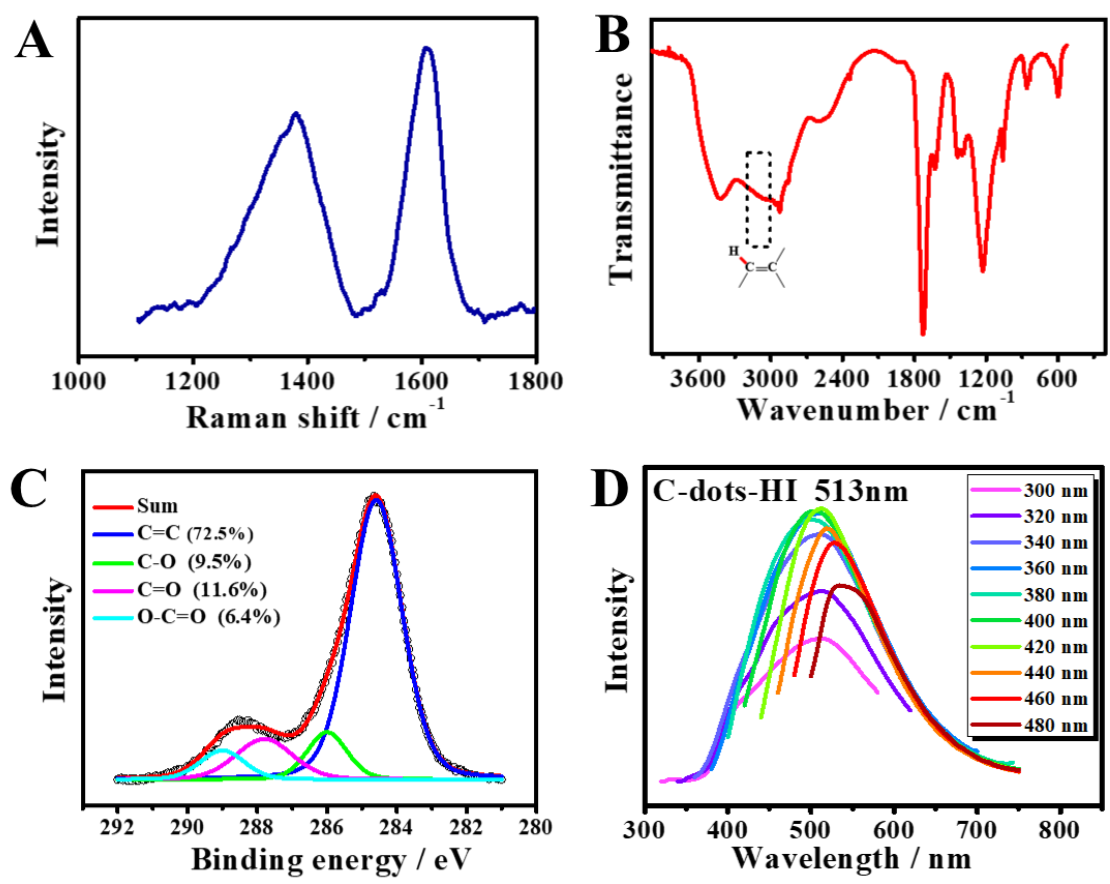

Figure S4. (A) Raman and (B) FT-IR spectra of C-dots-HI. (C) C 1s high-resolution XPS spectrum with identification of peaks by curve fitting, and (D) PL spectra under different excitation in $20 \mathrm{~nm}$ increments starting from 300 to $480 \mathrm{~nm}$ of C-dots-HI. 

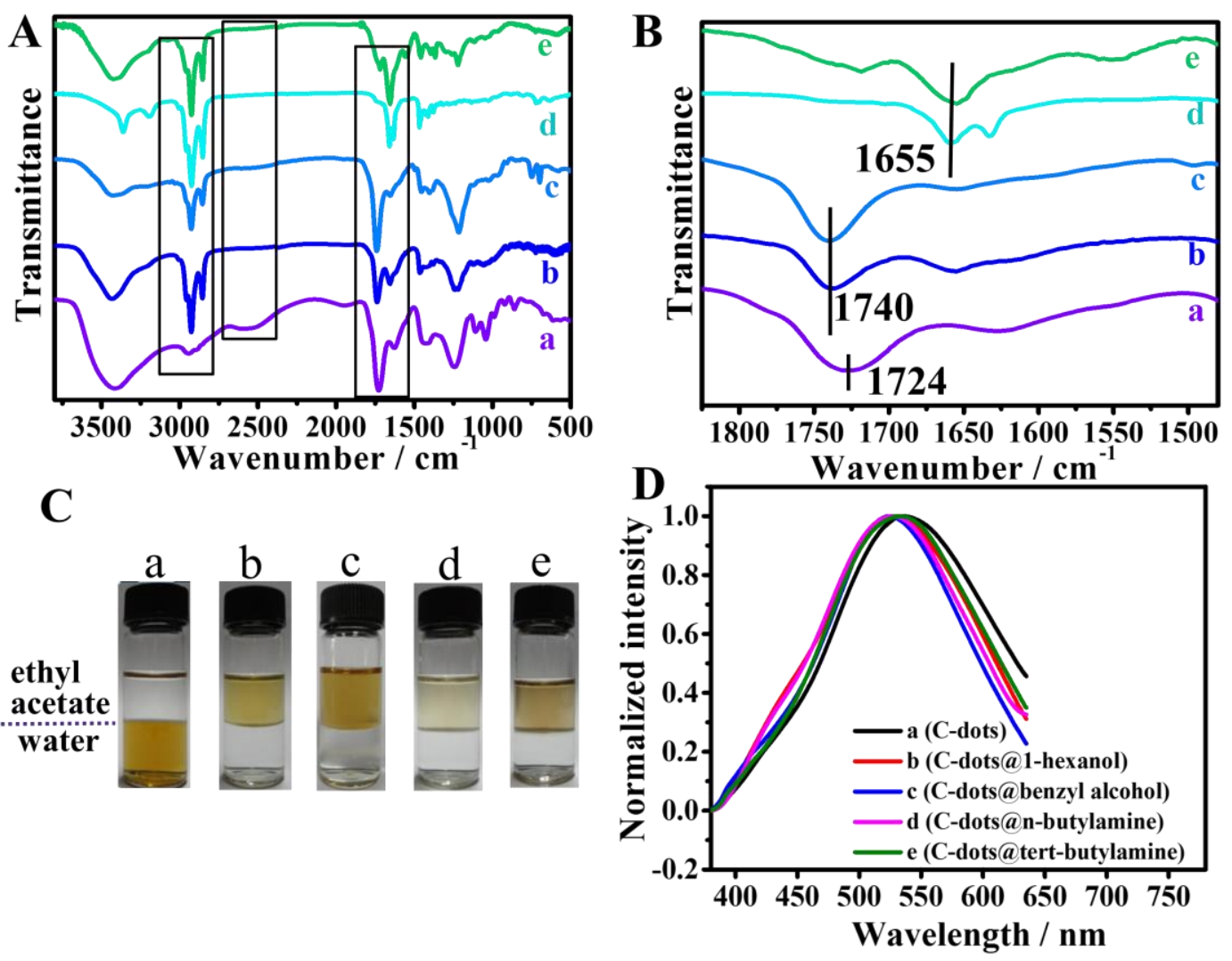

Figure S5. (A-B) FTIR spectra, (C) different distribution in the mixture of ethyl acetate and water, and (D) PL spectra of C-dots (a) before and after modification by (b) n-hexanol, (c) benzyl alcohol, (d) n-butylamine, (e) tert-butylamine.
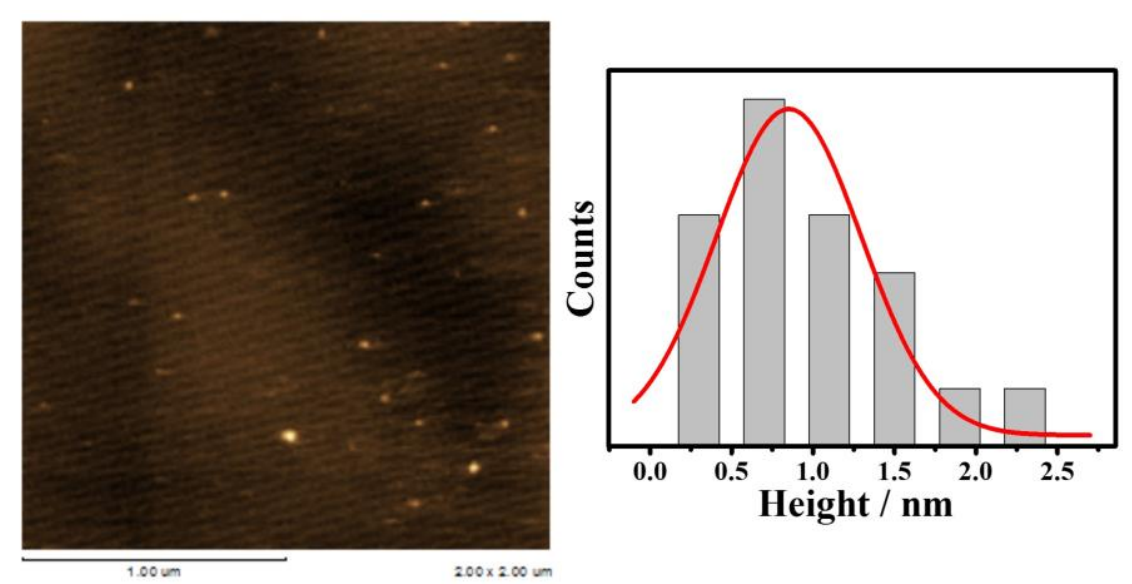

Figure S6. The AFM image and corresponding height distribution of C-dots 


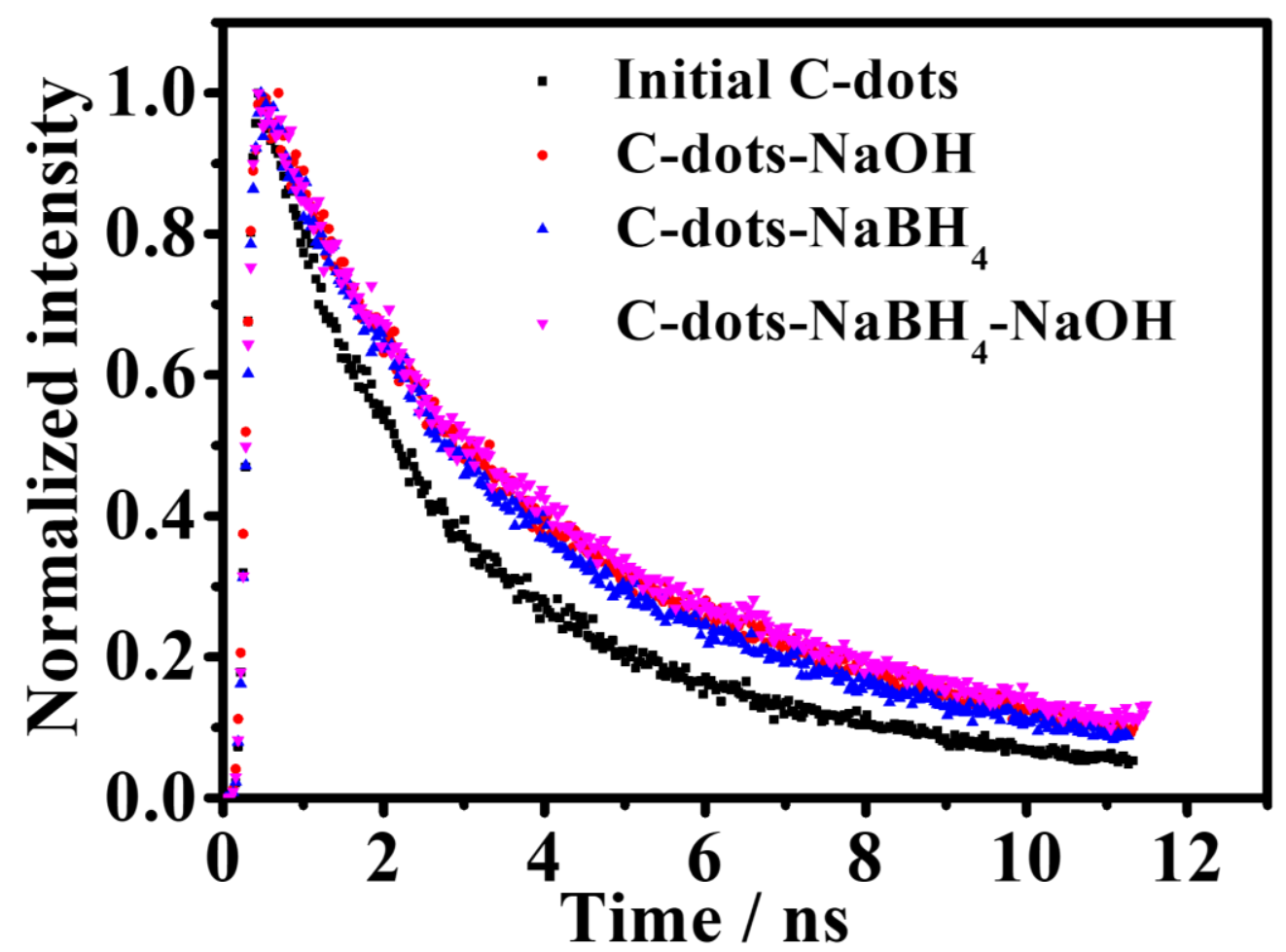

Figure S7. Time-resolved PL curves of the initial C-dots, C-dots-NaOH, C-dots- $\mathrm{NaBH}_{4}$, and C-dots- $\mathrm{NaBH}_{4}-\mathrm{NaOH}$ as indicated.

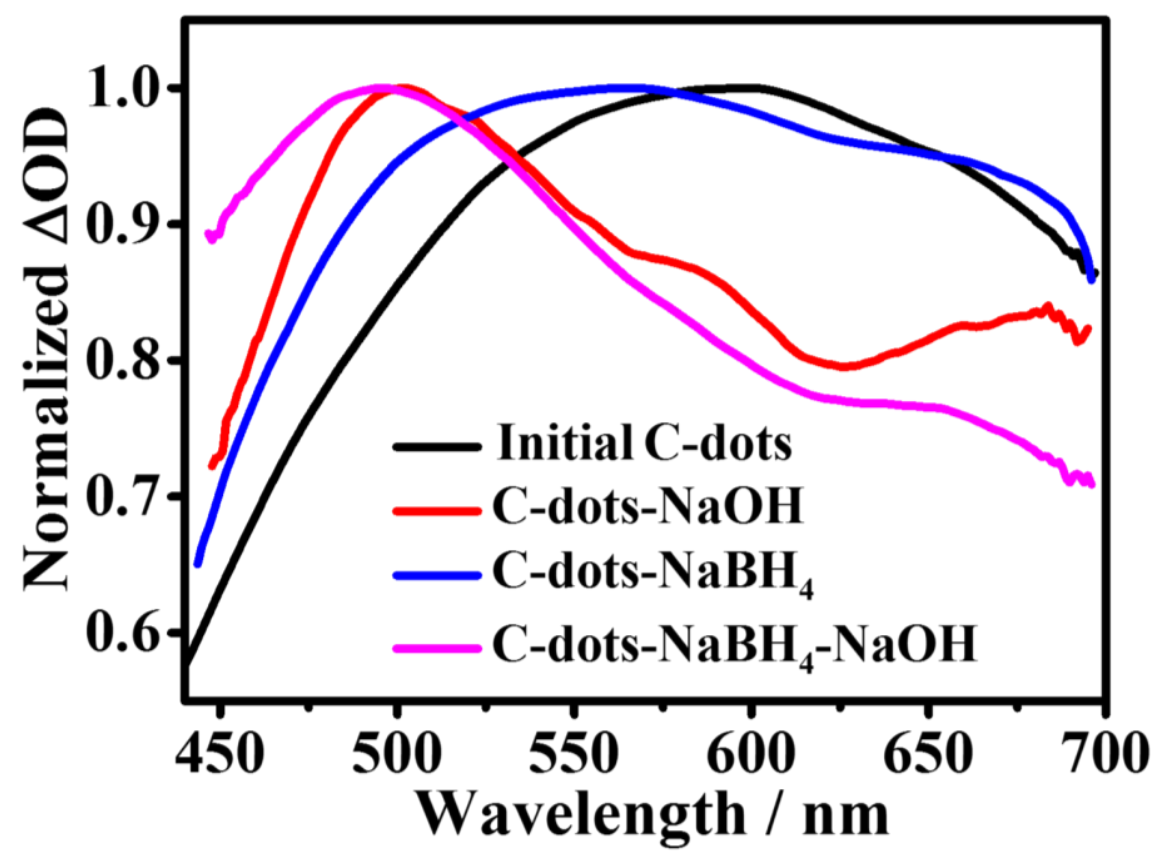

Figure S8. Typical transient absorption spectra (at 1 ps) of C-dots as indicated. 

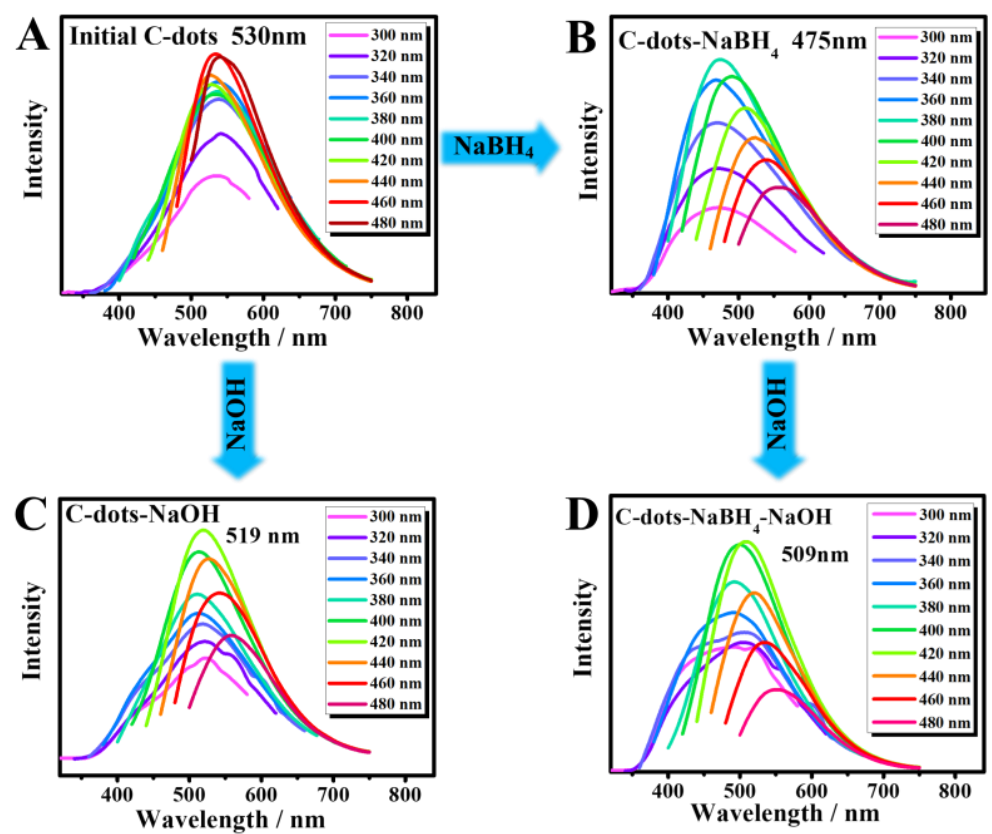

Figure S9. The PL spectra of C-dots as indicated under different excitation in $20 \mathrm{~nm}$ increments starting from 300 to $480 \mathrm{~nm}$.

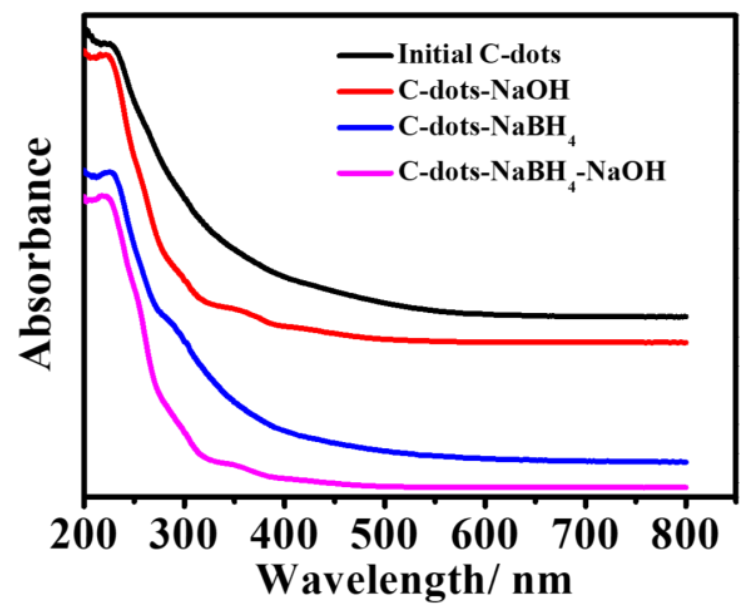

Figure S10. UV-Vis spectra of the initial C-dots, C-dots-NaOH, C-dots- $\mathrm{NaBH}_{4}$, and C-dots$\mathrm{NaBH}_{4}-\mathrm{NaOH}$.

As shown in Figure S10, after reduced by $\mathrm{NaHB}_{4}$, a new absorption peak at $280 \mathrm{~nm}$ appeared in the UV-vis spectrum of C-dots- $\mathrm{NaBH}_{4}$, which is in line with the previous report. ${ }^{9}$ A new peak at $350 \mathrm{~nm}$ attributed to the absorption of benzenoid band appeared in the UV-vis spectra of C-dots- $\mathrm{NaOH}$ and C-dots- $\mathrm{NaBH}_{4}-\mathrm{NaOH}$, confirming that the formation of larger $\pi$ electron system after hydro-thermal treatment in a $\mathrm{NaOH}$ solution. 
Table S1. Relative contents of different oxygen-containing functional groups and QYs of Cdots as indicated.

\begin{tabular}{lccccc}
\hline & $\mathrm{C}=\mathrm{C}$ & $\mathrm{C}=\mathrm{O}$ & $\mathrm{C}-\mathrm{O}$ & $-\mathrm{COO}$ & $\mathrm{QY}$ \\
\hline Initial C-dots & $63.6 \%$ & $19.3 \%$ & $11.0 \%$ & $6.2 \%$ & $2.0 \%$ \\
C-dots- $\mathrm{NaBH}_{4}$ & $64.7 \%$ & $16.3 \%$ & $13.7 \%$ & $5.3 \%$ & $4.0 \%$ \\
C-dots- $\mathrm{NaOH}$ & $68.8 \%$ & $13.6 \%$ & $7.8 \%$ & $9.8 \%$ & $12.6 \%$ \\
C-dots- $\mathrm{NaBH}_{4}-\mathrm{NaOH}$ & $72.6 \%$ & $10.6 \%$ & $8.4 \%$ & $8.4 \%$ & $14.1 \%$ \\
\hline
\end{tabular}

Table S2. Lifetimes of the initial C-dots, C-dots- $\mathrm{NaOH}$, C-dots- $\mathrm{NaBH}_{4}$, and C-dots- $\mathrm{NaBH}_{4}-$ $\mathrm{NaOH}$.

\begin{tabular}{llll}
\hline & $\tau_{1}(\mathrm{~ns})$ & $\tau_{2}(\mathrm{~ns})$ & $\tau_{\mathrm{avg}}(\mathrm{ns})$ \\
\hline Initial C-dots & $1.58(60 \%)$ & $5.39(40 \%)$ & 3.10 \\
C-dots- $\mathrm{NaBH}_{4}$ & $2.19(53 \%)$ & $6.34(47 \%)$ & 4.13 \\
C-dots-NaOH & $1.79(39 \%)$ & $5.89(61 \%)$ & 4.30 \\
C-dots- $\mathrm{NaBH} 4-\mathrm{NaOH}$ & $1.82(33 \%)$ & $5.83(67 \%)$ & 4.51 \\
\hline
\end{tabular}

References:

(1) Sun, H. COMPASS: An ab Initio Force-Field Optimized for Condensed-Phase ApplicationsOverview with Details on Alkane and Benzene Compounds, J. Phys. Chem. B. 1998, 102, 7338-7364.

(2) Stewart, J. J. P. Optimization of Parameters for Semiempirical Methods V: Modification of NDDO Approximations and Application to 70 Elements, J. Mol. Model. 2007, 13, 1173-1213.

(3) Klamt, A.; Schuurmann, G. COSMO: A New Approach to Dielectric Screening in Solvents with Explicit Expressions for the Screening Energy and its Gradient, J. Chem. Soc., Perkin Trans. 1993, 5, 799-805. 
(4) Foresman, J. B.; Headgordon, M.; Pople, J. A.; Frisch, M. J. Toward a systematic Molecular Orbital Theory for Excited States, J. Phys. Chem. 1992, 96, 135-149.

(5) Sun S.; Zhang S.; Liu K.; Wang Y.; Zhang B. The Geometry Relaxation and intersystem Crossing of Quaterthiophene Studied by Femtosecond Spectroscopy, Photochem. Photobio. Sci. 2015, 14, 853-858.

(6) Furniss, B. S.; Hannaford, A. J.; Smith, P. W.; Tatchell, A. R., Vogel's Textbook of Practical Organic Chemistry, Prarson Prentice Hall, London, 1989.

(7) Hola, K.; Bourlinos, A. B.; Kozak, O.; Berka, K.; Siskova, K. M.; Havrdova, M.; Tucek, J.; Safarova, K.; Otyepka, M.; Giannelis, E. P, Photoluminescence Effects of Graphitic Core Size and Surface Functional Groups in Carbon Dots: $\mathrm{COO}^{-}$Induced Red-Shift Emission, Carbon 2014, 70, 279-286.

(8) Liu, C.; Bao, L.; Tang, B.; Zhao, J.-Y.; Zhang, Z.-L.; Xiong, L.-H.; Hu, J.; Wu, L.-L.; Pang, D.-W. Fluorescence-Converging Carbon Nanodots-Hybridized Silica Nanosphere, Small 2016, 12, 4702-4706.

(9) Zheng, H. Z.; Wang, Q. L.; Long, Y. J.; Zhang, H. J.; Huang, X. X.; Zhu, R., Enhancing the Luminescence of Carbon Dots with a Reduction Pathway, Chem. Commun. 2011, 47, 10650-10652. 